\title{
Building and sharing multilingual speech resources, using ERIM generic platforms
}

\author{
Georges FAFIOTTE \\ GETA, CLIPS, IMAG-campus (UJF, Grenoble 1 Univ.) \\ 385 rue de la Bibliothèque, BP 53 \\ F-38041 Grenoble cedex 9 \\ France \\ georges.fafiotte@imag.fr
}

\begin{abstract}
In the framework of projects ChinFaDial and ERIM we have developed in recent years several platforms allowing to handle various aspects of bilingual spoken dialogues on the web -mainly, spontaneous speech corpus collection through distant human interpreting. Current development of the core ERIM-Interp and ERIM-Collect platforms now includes multimodal user interaction, integration of some machine aids (such as speech turn $\log$ s through speech recognition, or tentatively speech machine translation, both based on server-grounded market products), and next, online aids to speakers and/or interpreters.

First collected data should be made available on the web in fall 2004 (DistribDial) along with, as soon as available, a robust version of the collecting platform, in order to promote collaborative building, and sharing, of "raw" unannotated multilingual speech corpora.

A variant of the ERIM environment is to extend to distant $e$-training in interpreting, possibly creating situations which should in turn, in our view, foster larger-scale data collection and sharing in open access mode.
\end{abstract}

\section{Keywords}

Bilingual speech corpora, collaborative corpus collection, spontaneous dialogues, Web-based interpreting, multilingual communication, open-access resources, resource mutualization.

\section{Introduction}

Ongoing burst in the development of both portable telecommunications tools open to Internet transactions, and videoconferencing means, is creating rapid expansion of teleservicing and telebusiness applications with spontaneous dialogue, information inquiry, distant negotiation, etc. Multilingualism, now in spoken transaction as it has been in written one, appears as a key issue in distant communication, with sensitive questions, both in supporting the diversity of the native or origin language of conversing users (particularly within the opening European economic area), and in bringing some kind of balance between main "linguae francae" (common languages). Thus new stakes arise in enhancing distant web-based on-line interpreting services.

Meanwhile, Speech Machine Translation (SMT) steadily takes steps towards style spontaneity and multilingualism. In this context though, we face a notorious lack of large open-access corpora of bilingual spoken dialogues.

This led us to study, to model and propose a set of generic platforms, aiming at enhancing distant multilingual multimodal oral communication with full recording and collecting facilities, also addressing expectations from the MT systems engineering community.

The paper first looks over project motivation, then introduces the interpreting and collecting platforms presently available in the ERIM family, with current variants. It then reports on their first use in collecting domain-oriented spontaneously spoken French-Chinese dialogues. Finally we present ongoing or planned development, advocating for collaborative building and voluntary sharing of resulting multilingual resources.

\section{Motivations, early prototyping}

\subsection{Developing multilingual linguistic resources}

It is widely recognized that realistic and large corpora are key resources for building Speech Recognition (SR) and Speech MT systems. If the Web has recently been put to use as the largest possible corpus, modeling casual spontaneous spoken language requires transcribed speech corpora of hundreds of hours.

Speech translation systems thus need large parallel translation corpora of transcribed and aligned spontaneous utterances in dialogue context, ideally with complete sets of parse trees. However, few such corpora have been developed (by NEC, ATR 
and a few others), and these are not publicly available. Why not? Because these corpora are very expensive to transcribe once collected, and to annotate. After so much time has been spent in compiling a corpus, giving it away seems unreasonable.

Besides, a future research objective is to use collected corpora for studying and modeling real life spontaneous spoken language and dialogues, and possibly to investigate if and how specific linguistic traits can be expected depending on specific dialogue situations, translation process settings, or various multimodal interaction means.

For instance, two speakers in a bilingual dialogue may hear one another's original speech or not, they may use video or fixed images, etc. Their linguistic behavior is expected to vary accordingly: the number of clarification sub-dialogues may vary; third person use or indirect speech may be used more in the presence of a speech translation system than with a human interpreter; the use of deictic and anaphoric elements may turn out to depend on the use of visible markable objects on whiteboards, maps, images.

With these considerations in mind, we thus endeavoured to propose open-acces corpus resources - and therefore open-access collecting resources-, in order to ease collaborative building of "raw" unannotated multilingual translated speech corpora, likely taking advantage of new web-based interpreting situations or scenarios.

\subsection{Enhancing multilingual communication on the Web}

Some companies have already developed proprietary network-oriented interpreter's cubicles, which are the counterparts of existing fixed installations for interpreting in multilingual meetings (for example at the UN or EU). However, the associated code is not available for research.

Furthermore, our typical scenario is somewhat different from that of classical interpreting, where interpreters are available for the entire duration of the conversations. We rather allow two situations:

- "conference call": speakers establish a schedule, and book a time slot with an interpreter,

- "on demand interpretation": interlocutors try to converse using whatever knowledge they may have of their interlocutor's language, or of a third common language. When the language barrier impedes communication, they ask an available interpreter to jump in to help.

Apart from these practical motivations, we also wish to conduct experimental studies on the effect of combining multimodal resources on bilingual or multilingual conversations. Thus, full recording facilities were required anyhow.

\subsection{Pre-ERIM platforms}

Other studies of human "consecutive" interpretation have employed multimodal Wizard of Oz platforms (e.g. the EMMI plateform, that we experienced at ATR-ITL for bilingual pilotexperiments [Fafiotte \& Boitet, 1994] [Loken-Kim \& al., 1994]), or monolingual multi-Wizard architectures have been modelled in a multimodal setting (NEIMO [Coutaz \& al., 1996]). Thus our first objective was to produce a simulator of automatic speech translation systems in the same spirit, to gain experience and collect data.

We first built prototypes of a Speech MT Wizard of Oz simulator, Sim* [Fafiotte \& Zhai, 1999] (to be read as "Sim-Star", since being a parallel platform to the C-STAR II CLIPS environment). They were designed to run on the Internet, and were originally used on the intranet of CLIPSGETA. Network-based communications were handled by a client-server communication module developed in Tcl/Tk. Participants could see and hear each other and share an electronic whiteboard, using MBone resources.

The idea of using Wizard of $\mathrm{Oz}$ techniques in this context proved quite impractical, and thus was abandoned. Even if an acoustic filter was used to deform the interpreter's voice, participants perceived that a human was speaking. In the end, we realized that, even for true automatic high quality interpretation, there actually might well be a real human "warm body" in the loop anyway. Thus a realistic design for online interpretation could integrate both human and machine interpretation for "partially automatic" Speech MT.

The successive ERIM platforms have been implemented on this basis, in parallel at CLIPS with integrating the French language into multilingual Speech Machine Translation within C-STAR and NESPOLE! international projects. ERIM stands in French for Network-based Environment for Multimodal Interpreting.

\section{Distant human interpreting, as a collecting scheme for multilingual spoken dialogues}

\subsection{Context}

At CLIPS-GETA, one of the ultimate research goals in Speech MT is to build systems for automatic or partially automatic Speech Interpretation (i.e. "synergic" user-aided translation of speech). Much progress has been made in this area over the past twelve years. NEC produced the first speech translation demo in September 1992, within the tourist domain, but the most widely known coordinated research efforts to date include the C-STAR projects (now a 7-language 
international Consortium for Speech Translation Advanced Research) [http://www.c-star.org], the European NESPOLE! project [http://nespole.itc.it], the German Verbmobil [http://verbmobil.dfki.de] project, the US DARPA Communicator program with the Galaxy Communicator Software Infrastructure [http://fofoca.mitre.org/doc.html] [http://www.darpa.mil/ito/research/com/index.html] [http://www.sls.lcs.mit.edu/sls/whatwedo/architecture.html].

All have demonstrated platforms enhancing spontaneous speech processing in multilingual person-person or person-system communication, always in restricted domains. CLIPS is firmly involved in this action, while being in charge for integrating the French language in the C-STAR and NESPOLE! environments.

At the same time, we strongly believe that human interpreters will remain vital, both as irreplaceable suppliers of relevant nuances and as models for automatic or partially automatic systems.

Human interpreting, too, will inevitably be carried out through the Web and its raising applications. Thus we foresee a continuing need for research on Web-based interpreting, and for data collection of realistic general-purpose or domain-oriented Webbased interpreting sessions.

\subsection{Functionals of the ERIM human Interpreting platform}

The ERIM-Interp network-based environment consists of a central communication server, two speaker stations, one interpreter station (cf. Fig. 1), with a multimodality server (exchange of short typed messages, whiteboard with shared pictures or files, and shared pointing and marking). To avoid complex problems due to turn overlap, we have adopted a push-to-talk discipline up to now.

The current implementation of ERIM-Interp, in $\mathrm{Tcl} / \mathrm{Tk}$, is platform independent (and runs on Windows, MacOS, eventually Linux), and uses an adapted version of the CommSwitch written by CMU for the CSTAR-II project.

It is also flexible: the CommServer can be hosted on a dedicated station or on any user workstation, two speakers may share the same station (in a "visit" situation), the scenario can be extended to include more than two interlocutors, more than one interpreter (in "one-way" interpreting situations), and hence possibly more than two languages.

\section{Bilingual spontaneous speech collection}

\subsection{As the next step taken then, the ERIM Collecting platform}

We have then developed the ERIM-Collect variant, intended to collect corpora (cf. Fig. 1), moreover to enhance collaborative generation and use of bilingual speech corpora; namely to:

- collect only "raw" data (web-based spontaneous dialogues in any language pairs), as multimodal as possible - with no built-in annotation scheme intended yet,

- motivate volunteers to produce the data,

- induce volunteering by offering free service (on one of the ERIM variants described here), in exchange for free data (users should agree to "donate their speech to science"),

- distribute the data as freeware (via GPL licensing) on the Web, in a "re-playable" form: for each dialogue, descriptors indicate essential (anonymous) facts about the participants, along with the list of turns, indications of files, speakers, and time stamps for each turn,

- make it possible for other researchers to enrich the corpora by adding annotations in parallel files, again sharable through the web; they might use an extended version of the "Replay" facility (cf. Fig. 3), with consensus on a shared file structure and XML descriptors format,

- develop the collection platform so that it can itself be offered as freeware on the Web.

Accordingly, ERIM-Collect (currently 350 Kbytes of code in Tcl/Tk) was defined as an extension of ERIM-Interp:

- ERIM-Collect is language-independent,

- data is recorded locally during the dialogue; speech files are in PCM $22 \mathrm{kHz}-16$ bit-mono format,

- session and speech turns descriptor files are now in XML format,

- after the conversation, local descriptors and files are transferred then structured in corpus bases on a Collection Server,

- everything possible should be recorded: speech, short texts, whiteboard events, video, objects which the speakers refer to (e.g. file names and urls). In the current version 3 of ERIM-Collect, voice and short texts are collected; whiteboard actions and video are currently added.

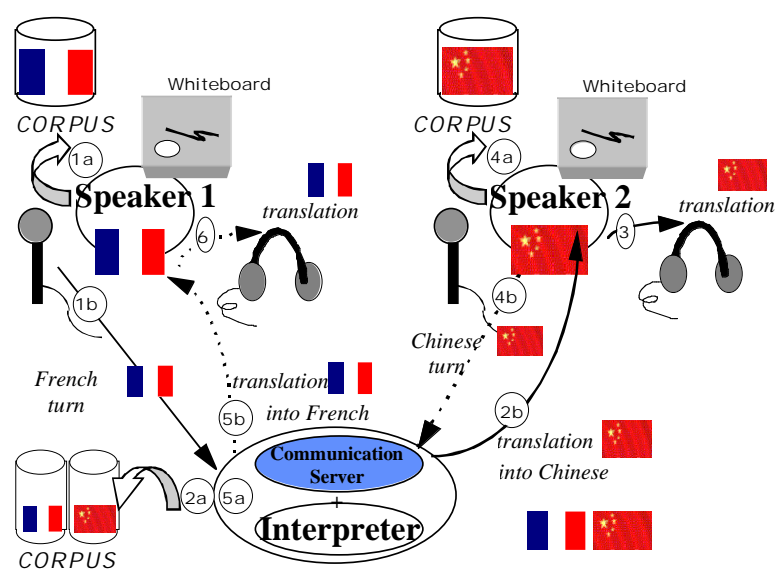

Figure 1: ERIM-Interp / ERIM-Collect 
We describe here (cf. Fig. 1) a basic exchange within a French-Chinese collection session. First (1), the French interlocutor takes a turn of one or more utterances. This turn (speech, descriptors) is recorded locally (1a), and transmitted (1b) to the Interpreter and the CommServer which broadcasts it across the virtual room established for the conversation. The interpreter listens to the turn and (2) translates it into Chinese. The translated turn is recorded locally (2a) and broadcast (2b). The Chinese participant listens to the translation (3) and then answers (4). Again, his answer is stored locally and broadcast (4a and $4 \mathrm{~b}$ ). The interpreter then translates it into French (5) and the translation is stored locally (5a) and broadcast (5b).

In order to create various experimental settings, we may unlock the reception of some messages for some participants. For instance in (1b) the French voice could be made audible for the Chinese participant.

As for playback of a previously recorded bilingual dialogue, a full reconstruction is available. Simplified visual tracking is provided as shown in Figure 3. One can extract monolingual versions of the dialogues.

A first version of the DistribDial / Replay component (and web site) for such replays has just been completed.
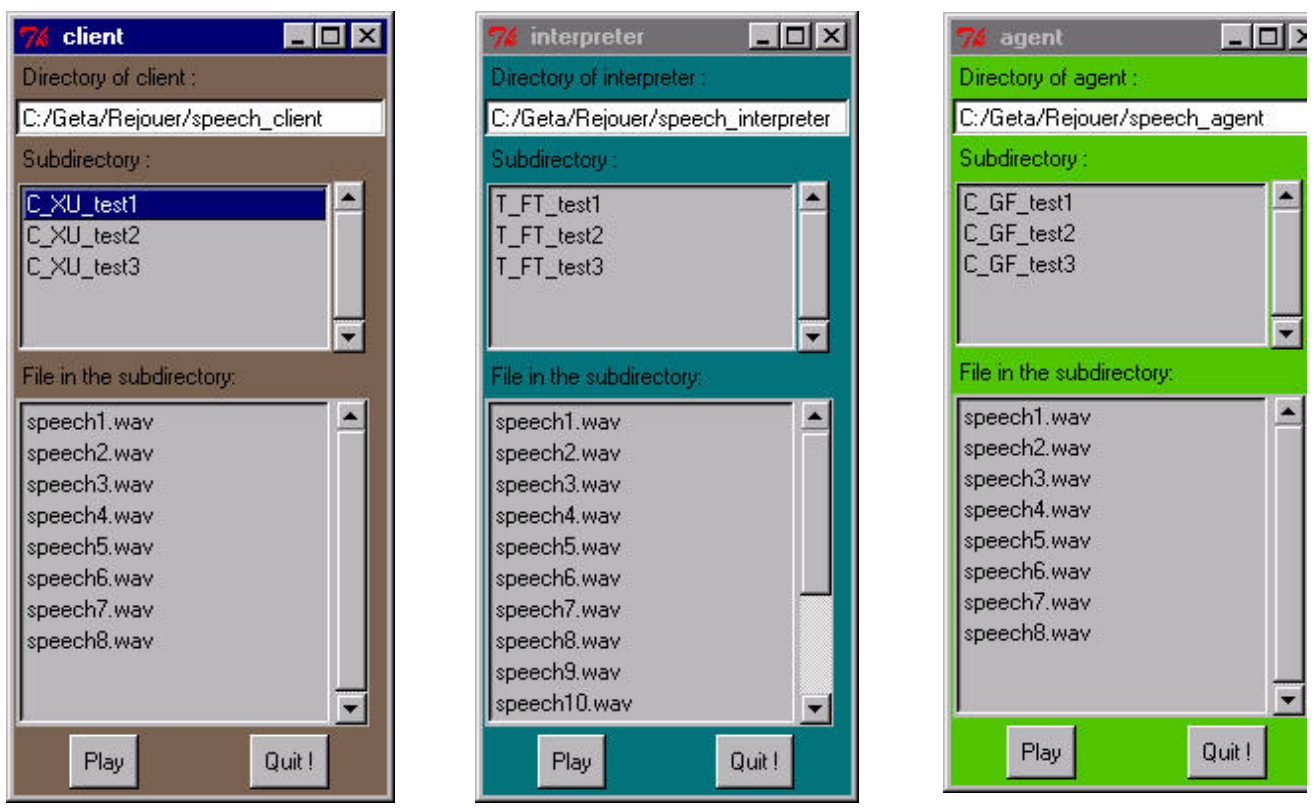

Figure 3: Playback of client, interpreter, and agent utterances

Successive versions of ERIM-Collect have been used for collecting first domain-oriented spontaneous speech corpora (hotel reservation) in Grenoble and Beijing (cf. 4.2).

\subsection{Providing online aid to interpreters and/or speakers}

In our "on demand interpretation" scenario, interpreters may be asked to jump from one conversation to another, and thus from one topic to another. This conversation switching is likely to be quite difficult, and stressful. Thus machine aids could be welcome: communication aids and language aids. We also envisage providing
Figure 2 shows the screen which is presented to a conversational partner, as presently prototyped for the ERIM-Collect platform.

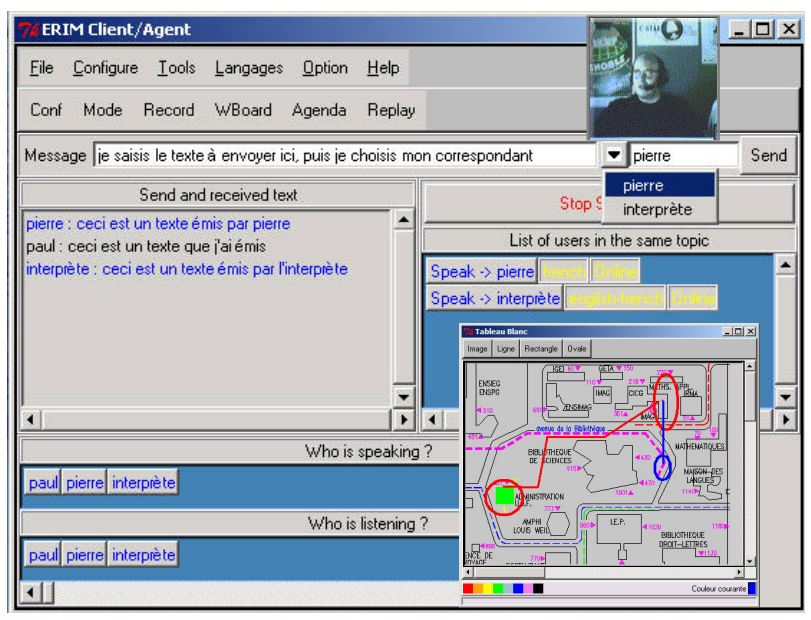

Figure 2: Speaker's screen

machine aids for the conversational partners, to help them do without interpreters so far as possible, if necessary.

The currently implemented "communication aids" include facilities to

- see and hear others (participants and interpreters),

- share data, possibly modifiable, markable, and "pointable" through the whiteboard,

- access an agenda for scheduling rendezvous.

Possible "language aids", to both the human interpreter and the speakers, are of three kinds:

- access to dictionaries via typed or voiced requests, and via automatic word spotting 
followed by filtering, dictionary look-up, and presentation in a dedicated window,

- speech recognition, to alleviate difficulties of oral understanding when not using the interpreter, and to produce a $\log$ of the conversation (which can additionally help an interpreter jump in), after possible reduction,

- fully or partially automatic speech translation.

At this time most communication aids have been implemented. The scheduling agenda is global for an ERIM site, but each user handles it through a personalized view (cf. Fig. 4).

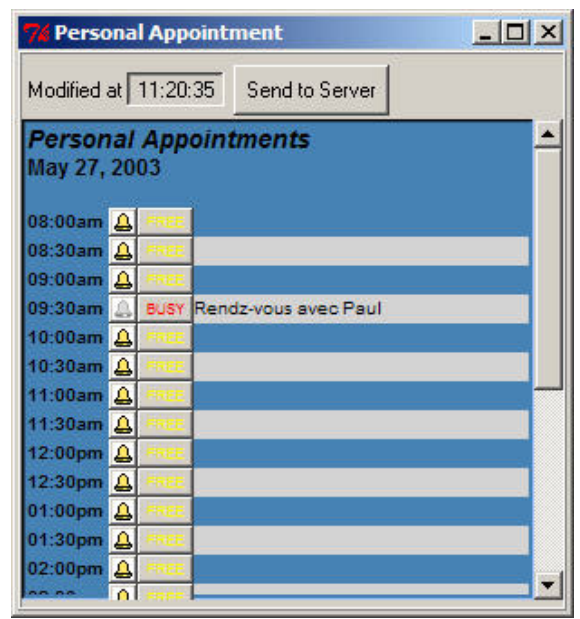

Figure 4: Window of user agenda

Language aids are the next step. An interface to existing free dictionary resources on the Papillon site [http://www.papillon-dictionary.org] should be added soon. A speech recognizer has been connected to the platform in another ERIM variant (the automatic interpretation pilot setup ERIMpaST). This Speech-To-Text facility could help as well to issue some draft transcripts during the dialogue.

\subsection{Adding partially automatic Speech MT}

An ERIM-paST (partially automated Speech Translation) platform is in progress at CLIPS in Grenoble, originally in cooperation with Spoken Translation Inc. (Berkeley). It aims at eventually providing some languge aids to speakers who "converse by themselves", and at allowing data recording of partially automatic interpreted dialogues (as a testing ground for Speech MT systems development, testing or tuning, at CLIPS). Experimentation with interactive disambiguation methods derived from the LIDIA project [Boitet \& Blanchon, 1994] is also expected.

The detailed description of this ERIM variant is beyond the scope of this paper. Briefly stated, the goal is here a generic modular integration, through plug-in, of Speech MT modules (speech recognizers, text-to-text translators, speech synthesizers), either research components (for their fine testing and tuning) or off-the-shelf products. Objective is to carry out comparative assessment of their results, or possibly contrastive evaluation with the human production of an interpreter "warm body".

A first version of ERIM-paST is currently being prototyped, while integrating server-based (Philips, Linguatec, Scansoft) market components.

\section{4 . First corpus collection, towards a collaborative building/sharing scheme}

\subsection{Platform assessment: distant collection}

Distant collection is also being tested, but in our first experiments Voice/IP still proved problematic when two turns overlapped. New efficient basic software and connection improvements are under evaluation. Record-then-send or record-whilesending (streaming) modes are available.

We may retain facilities for transmitting sound through phone lines. These might be used in operational contexts by telephone operators, such as Prosodie in France: since this company is also an Internet service provider, it can merge both tracks into a single communication.

Distant connection data is summarized in Figure 5.

\begin{tabular}{|c|c|c|c|c|}
\hline $\begin{array}{l}\text { Experiments } \\
\text { (Grades } \\
\text { from } 0 \text { to } 5 \text { ) }\end{array}$ & text & $\begin{array}{l}\text { voice: } \\
\text { record } \\
\text { then } \\
\text { send }\end{array}$ & $\begin{array}{c}\text { voice: } \\
\text { record \& } \\
\text { send } \\
\text { (streaming) }\end{array}$ & $\begin{array}{l}\text { voice: same } \\
\quad \text { with } \\
\text { overlapping }\end{array}$ \\
\hline Streaming & - & - & + & + \\
\hline $\begin{array}{l}\text { Connexion: } \\
\text { Internet }\end{array}$ & $\begin{array}{c}100 \\
\text { Mbit }\end{array}$ & $=$ & $=$ & $=$ \\
\hline $\begin{array}{l}\text { Reception } \\
\text { quality }\end{array}$ & 5 & 5 & 3 & 1 \\
\hline $\begin{array}{l}\text { Speed of } \\
\text { exchange }\end{array}$ & 5 & 2 & 4 & 5 \\
\hline Reliability & 5 & 5 & 4 & 1 \\
\hline $\begin{array}{l}\text { Special } \\
\text { problems / } \\
\text { phenomena }\end{array}$ & None & $\begin{array}{l}\text { User } \\
\text { wary } \\
\text { (too } \\
\text { slow) }\end{array}$ & $\begin{array}{c}\text { Some } \\
\text { micro-cuts, } \\
\text { but good } \\
\text { overall } \\
\text { quality }\end{array}$ & $\begin{array}{c}\text { Unusable, } \\
\text { bandwidth } \\
\text { too large }\end{array}$ \\
\hline
\end{tabular}

Figure 5: Oral communication over the web

\subsection{The ChinFaDial project, French-Chinese speech corpora}

The system has been used in the ChinFaDial project for collecting bilingual French-Chinese interpreted spontaneous spoken dialogues, in the hotel reservation domain. This 3-year project was funded by LIAMA, a joint French-Chinese laboratory under both French INRIA and Chinese 
CAS and MOST supervision. Our partner is the Chinese Information Processing group at NLPR (National Laboratory for Pattern Recognition), a research team within the Institute of Automation, Chinese Academy of Sciences (CAS-IA).

In ChinFaDial we have used intranets in Grenoble or in Beijing, with 3 participants using headsets, located in one or in 2 different buildings. It was possible for 2 speakers to share the same workstation, but we have mainly used the regular 3-station setting for the French-Chinese data collection. Some 10 hours of spontaneous translated spoken dialogues on "hotel information and reservation" have been recorded thus far. They produce about $43 \mathrm{kBits}$ per second.

Figure 5 shows a dialogue fragment transcription. We do not plan currently to transcribe or annotate corpora, but others will be very welcome to do so. Participants to this first data collection have been at this time:

\begin{tabular}{|l|c|c|c|}
\hline & Chinese & French & Total \\
\hline $\begin{array}{l}\text { Fr-Ch } \\
\text { Interpreters }\end{array}$ & 2 & 2 & 4 \\
\hline Interlocutors & 3 & 3 & 6 \\
\hline
\end{tabular}

There are 65 recorded dialogues with these characteristics:

\begin{tabular}{|l|c|c|c|}
\hline & Minimum & Average & Maximum \\
\hline Duration (sec) & 457 & 635 & 874 \\
\hline Number of turns & 28 & 52 & 78 \\
\hline Turn length(sec) & 4 & 12 & 57 \\
\hline
\end{tabular}

\section{顾客/Client (7) \\ 我在火车站; 火车站离你们旅馆不知道远不远、怎么走? \\ (Je suis à la gare, je ne sais pas comment me rendre à l'hotel à partir de la gare.)}

\section{Agent/代理 (7) \\ Alors c'est extrèmement simple,en sortant de la gare vous tournez à droite et c'est à 80 mètres en face de l'autre côté de la place.}

(很简单、如果你出了火车站以后向右转、只要走到 80 米左右。就是我们的旅馆。)

\section{所 客/Client (8) \\ 好谢谢那就一会儿见}

(Merci bien, alors à tout à l'heure)

Agent/代理 (8)

Merci, bonsoir Monsieur, à tout à l'heure.

(谢谢…谢谢、那么、这是…-会见)

Figure 5: Dialogue between a French hotel manager and a Chinese client (manual transcript)

\subsection{Ongoing developments, to promote collaborative corpus building}

A website with a small 'DistribDial' server has been prototyped to freely distribute the sound files and their descriptors, and a Replay module. Our goal is to extend it to allow other groups to contribute to the site whatever annotations they may have created, and to share them under the same conditions (GPL). They should only agree to share a common file base structure and a flexible XML desciptor format for each annotation file. Corpus collection in French-Chinese will extend. Further data collection using ERIM-Collect just started (spontaneous dialogues in French and Vietnamese, Tamil, Hindi), under support of AUF (University Agency for French-Speaking Communities), within the VTH-Fra.Dial project. We are also considering distributing an ERIMCollect "hardened" version on DistribDial, after strengthening robustness and usability, so that others can use it to do their own spoken dialogue collection.

\subsection{Planned e-Training extensions: use of the platform to involve volunteer interpreters}

Data collection being time-consuming all the same, our goal is not to do too much of it for its own sake, but to get it as byproduct of some "mutualized" use of the platform, in the open access mode.

Professional interpreters are unlikely to help on a non-profit basis, since interpreting is their livelihood. Improving junior interpreters or even advanced student interpreters, however, may find Web-based cooperation to be a good way of learning or perfecting their trade in real life situations.

We aim to induce volunteer interpreters or students of interpretation to translate bilingual dialogues online, by exchanging this on-line help for free use of our Web-based lab for $e$-training in interpretation.

We plan to develop an ERIM-Training variant platform, an $e$-training extension, with full recording of all speech interaction and any 
multimodal event. Actually we already simulated the functional architecture of it, using the current ERIM-Collect in a multi-interpreter setting.

Different scenarios and settings can be envisaged. For example, in a distant training or practice situation, for a student interpreter: the student might be alone, gaining experience, or might be with an instructor, who could supervise or take over.

At the 2008 Olympic Games in Beijing, as another example, good student interpreters could be asked to aid bilingual communication in exchange for academic credit, and free tickets. Assume, for instance, that a French speaker and a Chinese speaker want to converse. They could then go to a PC, activate ERIM-Interp with ERIM-Assist for French-Chinese, click on the icon of an available interpreter, and begin a mediated conversation, which would be recorded if participants agree while using the service free of charge.

\subsection{Building and sharing multilingual speech resources}

We advocate and expect ERIM-Collect, once proposed in an open-access mode on the Web, to be willingly and freely operated by other researchers, under an agreed collaborative framework to be set up, with minimal method and technical consent on collecting procedures and corpus characteristic profiles, in order to bring building and sharing of raw multilingual speech corpora to a more rapid expansion.

Collaborative annotation work could take place as well, again with simple agreed procedures on content and descriptor files formats, and on a public use scheme.

Such tools, and their open use, could as well underlie valuable action towards supportive protection of "smaller languages", among others minor European languages, while for instance fostering distant learning of interpreting, and while easing the use of low-cost or even free interpreting facilities over the net.

\section{Unification of ERIM platform variants}

Work is now beginning on the integration of the different platforms presented here into one single multifunctional ERIMM system [Fafiotte \& Boitet, 2003], for enhancing free multilingual multimodal network-based communication with distant interpreting and corpus collection.

Numerous technical issues arise in this effort. For instance, it is not immediately clear how the CommServer will accommodate server-based interactive lexical disambiguation during translation; or how to secure efficient streaming data transmission in a multicast scheme. Even so, the platform independence and plug-and-play generic architecture of ERIM set components make this integration effort quite realistic, in spite of the number and diversity of functions to be integrated.

\section{Conclusion}

We have presented several platforms developed in the long-range ERIM project. Each platform can aid in the study of spontaneous cross-lingual communication on the Web. The core platform is ERIM-Interp for Web-based human interpretation. ERIM-Collect is a deliberate development of the latter, dedicated to multilingual "raw" speech corpus building, and intended to alleviate the current scarcity of data — particularly open data-, and which can also support the construction of speech translation systems.

ERIM-Assist will add various machine aids for interpreters and conversational partners, while ERIM-paST (only briefly mentioned here) includes components for partially automatic speech translation.

We then reported on a first collection of spontaneous bilingual interpreted spoken dialogues for French-Chinese. This data, along with the collecting framework itself, will be distributed in the near future on the Web as shareware or GPLware, under a DistribDial component.

We are looking for funding to create ERIMTraining - a further extension of ERIM-Interpwhich could serve as a valuable "Web-based language lab for interpreting" for distant $e$-training, while also providing new facilities for language learning.

We plan to continue research in the ERIM framework by collecting and distributing more data concerning more languages (Vietnamese, Tamil, Hindi to French). Data collection should be enhanced by a unified version of ERIM, offering all the functionalities of the platform variants.

More specifically, we hope that junior interpreters or advanced students in interpreting will volunteer to interpret and to practice with ERIM-Training, while users would agree to give their dialogues to science in exchange of using ERIM-Interp for free.

\section{Acknowledgements}

This work has been supported by CLIPS-IMAG (UJF University Grenoble 1, CNRS, INPG) and funded in part by the LIAMA French-Chinese Laboratory (ChinFaDial project), and by the Rhône-Alpes Region (ERIM project). Corpus collecting action is currently supported by AUFLTT (University Agency for French-Speaking Communities, VTH-Fra.Dial project).

Our thanks go to Zhai JianShe (Nanjing 
University, China) for early prototyping, to Julien Lamboley (at INSA, Lyon, France) for platform development, to members of the GETA and NLPR/CASIA-Beijing teams and to Brigitte Meillon at CLIPS-MultiCom, for their participation in data collection and related experiments.

\section{References}

Boitet C. \& Blanchon H., 1994. Multilingual Dialogue-Based MT for Monolingual Authors: the LIDIA Project and a First Mockup. Machine Translation 9/2/94, pp. 99-132.

Coutaz J., Salber D., Carraux E. \& Portolan N., 1996. NEIMO, a Multiwork station Usability Lab for Observing and Analyzing Multimodal Interaction. Proc. CHI'96 companion.

Fafiotte G. \& Boitet C., 1994. Report on first EMMI Experiments for the MIDDIM project in the context of Interpreting Telecommunications. MIDDIM report TR-IT-0074 GETA-IMAG \& ATR-ITL, Aug. 94, 11 p.

Fafiotte G. \& Boitet C., 2003. ERIMM, a platform for supporting and collecting multimodal spontaneous bilingual dialogues. IEEE NLPKE2003, Beijing, 26-29/10/03, 6 p.
Fafiotte G. \& Zhai J.-S., 1999. A Network-based Simulator for Speech Translation. Proc. NPLRS'99, Beijing, 5-7/11/99, B. Yuan, T. Huang \& X. Tang ed., pp. 511-514.

Furuse O., Sobashima Y., Takezama T. \& Uratani N., 1994. Bilingual corpus for speech translation. Proc. AAAI-94 Workshop on Integration of Natural Language and Speech Processing, Seattle, Washington, USA, 31/71/8/94, ATR Interpreting Telecommunications.

Loken-Kim K.-H., Yato F. \& Morimoto T., 1994. A Simulation Environment for Multimodal Interpreting Telecommunications. Proc. IPSJAV workshop, March 94, 5 p.

<url> C-STAR. http://www.c-star.org <url> DARPA sites.

http://www.darpa.mil/ito/research/com/index.html, http://fofoca.mitre.org/doc.html

<url> GALAXY system architecture site. http://www.sls.lcs.mit.edu/sls/whatwedo/ architecture.html

<url> site web NESPOLE! http://nespole.itc.it $<$ url > site web PAPILLON.

http://www.papillon-dictionary.org

[<url> VERBMOBIL site. http://verbmobil.dfki.de 